\title{
School to go: Neues Lernen im Social-Media-Stil
}

\author{
Jannick Eckle • Anne Jungfleisch • Daniel Stattkus $(\mathbb{D} \cdot$ Novica Zarvić • \\ Julia Knopf • Oliver Thomas
}

Eingegangen: 4. Juni 2021 / Angenommen: 6. September 2021 / Online publiziert: 14. Oktober 2021

(C) Der/die Autor(en) 2021

Zusammenfassung Mit dem pandemiebedingten ersten Lockdown im März 2020 hat das digitale Lernen einen enormen Aufschwung erfahren. Mit der Initiative „School to go“" wird in Zeiten bundesweiter Schulschließungen ein relevanter Beitrag zur digitalen Bildung für Schülerinnen und Schüler, Eltern, Lehrkräfte und die interessierte Öffentlichkeit geleistet. Auf der gleichnamigen Plattform (www. schooltogo.de) werden digitale Lernangebote für Kinder, Jugendliche sowie junge Erwachsene gebündelt. Ferner finden sich dort Blogbeiträge zu Trendthemen der digitalen Bildung: Wie können digitale und didaktisch motivierte Raumkonzepte zu

Jannick Eckle

Didactic Innovations GmbH, 66123 Saarbrücken, Deutschland

E-Mail: jannick.eckle@didactic-innovations.de

Anne Jungfleisch · Julia Knopf

Forschungsinstitut Bildung Digital, Universität des Saarlandes, 66123 Saarbrücken, Deutschland

Anne Jungfleisch

E-Mail: anne.jungfleisch@uni-saarland.de

Julia Knopf

E-Mail: julia.knopf@mx.uni-saarland.de

Daniel Stattkus $(\bowtie) \cdot$ Novica Zarvić · Oliver Thomas

Smart Enterprise Engineering, Deutsches Forschungszentrum für Künstliche Intelligenz (DFKI)

GmbH, Parkstraße 40, 49080 Osnabrück, Deutschland

E-Mail: daniel.stattkus@dfki.de

Novica Zarvić

E-Mail: novica.zarvic@dfki.de

Oliver Thomas

E-Mail: oliver.thomas@dfki.de

Oliver Thomas

Fachgebiet Informationsmanagement und Wirtschaftsinformatik, Universität Osnabrück,

Parkstraße 40, 49080 Osnabrück, Deutschland 
einem verbesserten Lernen beitragen und welche Bedeutung hat Gamification für die Lernmotivation der Schülerinnen und Schüler? Derzeit befinden sich auf „School to go" bereits über 1000 innovative Lernformate (Stand Mai 2021) für verschiedene Fächer, die sich an Schülerinnen und Schüler aller Alters- und Jahrgangsstufen richten. Der vorliegende Beitrag zeigt, wie neues Lernen im Zeitalter der Digitalisierung gelingt und wie hierbei Didaktik und Wirtschaftsinformatik in Einklang gebracht werden können, um nachhaltige Lernerlebnisse zu ermöglichen.

Schlüsselwörter Lernen · E-Learning · Social Media · Plattform ·

Personalisierung $\cdot$ Empfehlungssysteme

\section{School to Go: New Learning in a Social Media Style}

Abstract With the pandemic-related first lockdown in March 2020, digital learning has experienced a tremendous boost. In times of nationwide school closures, the "School to go" initiative makes a relevant contribution to digital education for students, parents, teachers and the interested public. The platform of the same name (www.schooltogo.de) bundles digital learning opportunities for children, young people and young adults. It also features blog posts on trend topics in digital education: How can digital and didactically motivated spatial concepts contribute to improved learning and what significance does gamification have for students' motivation to learn? Currently, there are already over 1000 innovative learning formats (as of May 2021) for various subjects on "School to go", aimed at students of all ages and grades. This article shows how new learning succeeds in the age of digitization and how didactics and business informatics should be harmonized to provide sustainable learning experiences.

Keywords Learning · E-Learning - Social Media · Platform · Personalization · Recommender systems

\section{Einleitung}

Seit März 2020 hält die Corona-Pandemie die ganze Welt in Aufruhr. Weitreichende Auswirkungen sind seitdem vor allem auch im Bildungssektor deutlich spürbar. Während Universitäten und Fachhochschulen auf digitale Präsenzveranstaltungen umstellten, wurden Kitas und Schulen durch die Schließungen kurzfristig dazu veranlasst, ihr bisheriges Bildungskonzept $\mathrm{zu}$ überdenken und neu $\mathrm{zu}$ gestalten. Die Bereitstellung der schulischen Lernangebote erfolgte dabei uneinheitlich über unterschiedliche Kanäle. Als besonders etabliert galt jedoch die Übermittlung der Materialien über einen klassischen E-Mail-Verteiler: Vor allem digitale Scans von ursprünglich nicht digitalen Materialien (z.B. Fotografien von Tafelbildern) oder aber Verweise zu Aufgabenstellungen in analogen Lehrbüchern zählen zu denjenigen Formaten, die häufig an Lernende versandt wurden. Zahlreiche Studien zeigen: Trotz des hohen Engagements fühlten sich knapp ein Viertel aller befragten Lehrkräfte bei der Planung und Gestaltung ihrer Lernangebote auf sich allein gestellt 
(Eickelmann und Drossel 2020). Hier setzt die Initiative „School to go“ an, die von Didaktik- und KI-Experten des FoBiD (Forschungsinstitut Bildung Digital), des DFKI (Deutsches Forschungszentrum für Künstliche Intelligenz) und der Didactic Innovations GmbH ins Leben gerufen wurde. „School to go“ stellt Lernenden neben den von den Schulen zur Verfügung gestellten Arbeitsplänen und Aufgaben motivierende und passgenaue Angebote für das Distanzlernen zur Verfügung und bündelt diese auf der gleichnamigen Plattform. Die Lernangebote durchlaufen eine umfassende inhaltliche, didaktische und technologische Qualitätsprüfung (siehe 4.1), bevor sie schließlich von den Lehrenden, Lernenden und der interessierten Öffentlichkeit unter www.schooltogo.de kostenlos genutzt werden können. Auf der übersichtlich gestalteten Plattform, die aufgrund des Aufbaus und Kachel-Designs an gängige Social-Media-Kanäle wie Pinterest und Instagram erinnert, können User von „School to go“ fokussiert nach Jahrgangsstufen (von Klasse 01/02 bis hin zu Klasse 11/12) und Lernbereichen (z. B. Deutsch, Fremdsprachen, MINT und LernMix) filtern und so aus einer Vielzahl innovativer Lernformate wählen. Diese reichen von interaktiven Videos zu verschiedenen lehrplanrelevanten Themen bis hin zu motivierenden Selbstlernmodulen, mit denen die Lernenden z. B. ihre Präsentationskompetenzen weiterentwickeln können (siehe 4.2). Allen Lernangeboten ist gemeinsam: Sie zeichnen sich durch einen hohen Aufforderungscharakter aus und orientieren sich an den tatsächlichen Interessen der Lernenden. Durch die Integration dieser Materialien und Medien in eine Social-Media-ähnliche Lernumgebung, die an den Bedarfen der User angepasst ist und sich an ihren privaten Gewohnheiten in Social-Media-Kanälen orientiert, lässt sich intrinsische Lernmotivation auch für das selbstregulierte Lernen auf Distanz aufbauen.

\section{New Learning meets Social Media}

\subsection{Umgang mit Social Media}

Unter dem Begriff Social Media wird die „Vernetzung von Benutzern und deren Kommunikation und Kooperation über das Internet“" verstanden (Bendel 2021). Demnach werden unter dem Begriff nicht neue Technologien und einzelne Plattformen wie Facebook oder Instagram zusammengefasst, sondern vielmehr das, wozu Menschen die Plattformen nutzen: zum sozialen Austausch. Dies ist einer der Gründe dafür, warum Social Media so beliebt ist. Laut einer Auswertung des statistischen Bundesamtes (2020) nutzen 89\% der 16- bis 24-Jährigen Social Media im Internet für private Kommunikation. Unter den 25- bis 44-Jährigen beträgt der Anteil $73 \%$ und auch unter den 45- bis 64-Jährigen nutzt rund jeder Zweite soziale Netzwerke zur Kommunikation mit Freunden, Familie und Bekannten (Statistisches Bundesamt 2020). Am meisten genutzt wird zurzeit Instagram, im Jahr 2019 war es noch Facebook. So bestätigt die ARD/ZDF-Onlinestudie (2020), dass $15 \%$ der Bevölkerung Deutschlands täglich Instagram nutzt - Tendenz steigend. Neben der Chatmöglichkeit treten Nutzende durch das Teilen von Bildern und Videos mit anderen Nutzenden in Kontakt. Doch auch Marken im Lifestyle-Bereich profitieren auf Instagram vom sogenannten Influencer-Marketing. Hierbei handelt es sich um ,die 
Planung, Steuerung und Kontrolle des gezielten Einsatzes von Social-Media-Meinungsführern und Multiplikatoren, um durch deren Empfehlungen die Wertigkeit von Markenbotschaften zu steigern und das Kaufverhalten der Zielgruppe positiv zu beeinflussen“ (Deges 2018, S. 35). Das „Prinzip Meinungsmacher“, das hinter dem Influencer-Marketing steckt, ist nicht neu - bereits im 18. Jahrhundert begann der Unternehmer Josiah Wedgwood mit der britischen Königsfamilie für seine Produkte zu werben (Jahnke 2021). Es kann also an dieser Stelle festgehalten werden, dass Instagram mehr bietet als den Austausch und die Kommunikation mit Familien, Freunden und Bekannten.

Trotz alledem gering vertreten auf der Plattform ist die Generation Z. Darunter fallen alle Personen, die zwischen 1995 und 2010 geboren wurden (Kleinjohann und Reinecke 2020). Laut ARD/ZDF-Onlinestudie (2020) ist TikTok die Social-MediaPlattform der Generation Z. Das heißt, dass Nutzende dazu angeregt werden, ,,vorhandene Formate, Vorlagen oder Ideen zu nutzen und neu zu erstellen, um eigene Persönlichkeiten oder Ideen auszudrücken“ (Hubspot 2021, S. 4). Der Content wird über einen Feed anderen Nutzenden ausgespielt, ohne dass man selbst danach suchen muss. Das Besondere an TikTok: Anders als bei Instagram werden Nutzende nicht nur für ihre Reichweite, sondern vor allem für guten Content belohnt. Dadurch ist bei der Content-Erstellung ein hohes Maß an Kreativität gefragt. Durch die Duett-Funktion von TikTok erfahren Nutzende zudem die Möglichkeit, sich nach dem Prinzip ,Learning-by-Doing“ Kompetenzen anzueignen, indem Videos von Experten durch Nutzende nachgestellt und anschließend als Duett veröffentlicht werden. Es existieren derweil zahlreiche TikTok-Kanäle, die Lernvideos zu verschiedenen Fachbereichen (z. B. Mathematik, Deutsch als Zweitsprache, Sprachenlernen) produzieren.

TikTok nutzt bereits Eigenschaften und Funktionalitäten der Plattform für das Lernen. Inwiefern weitere Eigenschaften von Social Media wie Individualisierung, Personalisierung und Kreativität auch auf weiteren Plattformen für das digitale Lehren und Lernen genutzt werden können und wie diese mit der Didaktik des New Learning in Einklang gebrachten werden, wird nachfolgend näher erläutert.

\subsection{Old School Learning vs. New Learning}

Im Gegensatz zur wachsenden Beliebtheit von Social Media gestaltet sich das digitale Lehren und Lernen während des bundesweiten Lockdowns in der CoronaPandemie vergleichsweise schwierig (siehe Einleitung). Zurückzuführen ist dies auf die Hürden und Probleme des digitalen Lehrens und Lernens. Lehrkräfte übertragen Konzepte des klassischen Lehrens und Lernens im analogen Klassenzimmer auf das Lehren und Lernen online. So werden Tafelbilder nicht im Klassenraum entwickelt, sondern abfotografiert und im Learning-Management-System hochgeladen, Arbeitsblätter werden nicht im Klassenraum ausgeteilt und in Partnerarbeit bearbeitet, sondern lediglich per Mail versandt. Das Resultat: Lehrende und Lernende sind überfordert und fühlen sich allein gelassen (Eickelmann und Drossel 2020). Experten sprechen von einem „New Learning“, das an die Stelle des „Old School Learning“" treten muss. Graf und Schmitz (2020, S. 80) definieren hierbei New Learning wie folgt: 
Abb. 1 Anforderungen an das New Learning

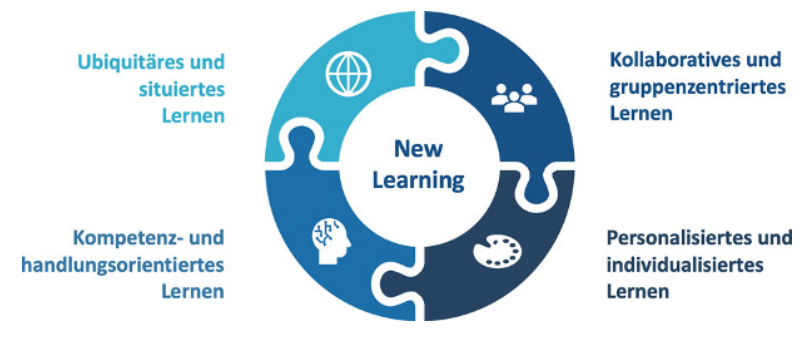

New Learning basiert auf Frithjof Bergmanns New-Work-Konzept und hat die Selbst- und Potenzialentfaltung des Individuums zum Ziel. New Learning bezeichnet Lernen, das vom Lernenden als sinnhaft erlebt wird und die Teilhabe an der Gemeinschaft ermöglicht. Die Lernprozesse sind geprägt von Selbstbestimmung, Autonomie und dem Streben nach Wirksamkeit. Dabei gilt, dass die Lerner ein hohes Maß an Selbstverantwortung und die Zugehörigkeit zur (Lern-)Gemeinschaft erleben.

Es stellt sich die Frage, wie New Learning erreicht werden kann. Zunächst wird bei Betrachtung der Studienergebnisse während der Corona-Pandemie (siehe Einleitung) deutlich, dass es nicht möglich ist, die Didaktik der klassisch analogen Lehr-Lern-Settings in den digitalen Bereich zu übertragen. Die Didaktik stellt neue Anforderungen an das New Learning (siehe Abb. 1).

Zum einen sollte digitales Lehren und Lernen ubiquitär stattfinden und dadurch ein unsichtbarer Teil des Alltags werden (Kartes et al. 2020) - in etwa so, wie Social Media es bereits für den Großteil der Bevölkerung ist. Die Informationsflut muss jedoch beim New Learning didaktisch aufbereitet werden, um strukturiertes Lernen zu ermöglichen. Innovative und didaktische Lernformate sollten hierbei zum Einsatz kommen. Das situierte Lernen bringt zum anderen zweierlei Sichtweisen zum Ausdruck: Lernen soll demnach stets an die Situation gebunden sein, in der sich der Lernende befindet - vorausgesetzt, die Lernsituation ist als solche relevant für den Lernprozess. Weiterhin wird davon ausgegangen, dass sich der Lernprozess und damit die kognitiven Prozesse, ,nicht allein im Individuum abspielen, sondern vor allem im Austausch mit anderen“ (Spieler 2006). Lernen wird damit ,nicht als individueller Fortschritt verstanden, sondern als Hineinwachsen in eine community of practice“ (Spieler 2006). Sowohl der Community-Gedanke als auch das Prinzip des gegenseitigen Wissensaustausch könnten somit aus der Social-Media-Welt auf das digitale Lehren und Lernen übertragen werden. Im Sinne des Influencer-Marketings ist es beispielsweise denkbar, dass Nutzende ihr Wissen effektiv an Interessierte vermitteln (Kartes et al. 2020).

Eng im Bezug zum situativen Lernen steht das kollaborative und gruppenzentrierte Lernen. Vor allem im digitalen Bereich ist zwar der Kommunikationsaspekt in Form von Chat- und Gruppenfunktionen hoch vertreten. Trotzdem erscheinen Nutzende in Learning-Management-Systemen (LMS) oder auf den Social-MediaPlattformen oft anonym. Kollaborative Lernformen sollen Abhilfe schaffen und dazu beitragen, dass Lernen im konstruktiven Austausch stattfindet. Es existieren bereits 
zahlreiche Tools, die das Lernen in der Gruppe ortsunabhängig und in Echtzeit ermöglichen. Ein Beispiel hierfür ist die kostenlose digitale Pinnwand (,Padlet“"), auf welcher Multimediaformate abgelegt und im Social-Media-Stil gelikt und kommentiert werden können (siehe www.padlet.com).

Eine weitere Anforderung, die die Didaktik an das New Learning stellt, ist die Kompetenz- und Handlungsorientierung. Mit dem in den letzten Jahren vollzogenen Wandel von der produktorientierten Didaktik hin zur prozessorientierten Didaktik kommt dem Kompetenzerwerb des Lernenden durch handlungsorientiertes Lernen auch im digitalen Bereich zusätzlich Bedeutung zu. Der Lernende steht mit seinem Handeln im Mittelpunkt des Geschehens. Damit dies gelingt, brauchen Lehrende und Lernende kontinuierliches und regelmäßiges Feedback - sowohl zu Inhalten als auch zum eigenen Lernprozess. Im digitalen Bereich bedarf es hierfür Learning Analytics und neuer Technologien wie Künstliche Intelligenz. Lernplattformen müssen in der Lage sein, Lerner- und Lehrerdaten zu sammeln und entsprechend zu analysieren (siehe Kap. 3).

Zur prozessorientierten Didaktik zählen neben der Kompetenz- und Handlungsorientierung auch die Individualisierung und Personalisierung des Lernens. Beide Prinzipien gehen von dem Ansatz aus, dass der Lerner mit seinen unterschiedlichen Lernvoraussetzungen, Bedürfnissen und Interessen als Individuum angesehen wird. Beim individualisierten Lernen sind die Lernziele, die erreicht werden sollen, für alle Lernenden die gleichen. Die Art und Weise, wie diese Ziele erreicht werden, ist jedoch in Bezug auf Lerntempo, -materialien und -formate von Lernendem zu Lernendem unterschiedlich. Blended-Learning-Kurse oder individualisierte Lernpfade sind Möglichkeiten für individualisiertes Lernen im Bereich des digitalen Lehren und Lernens. Die Personalisierung des Lernens unterscheidet sich von der Individualisierung dadurch, dass die Lernziele nicht allgemein festgelegt sind, sondern dass sowohl Lernziele als auch die entsprechenden Lerninhalte auf die Bedürfnisse des Lernenden abgestimmt werden. Hierfür bedarf es einer hohen Selbstlernkompetenz des Lernenden. Der Lehrende steht an dieser Stelle als Trainer und Mentor zur Verfügung (Department of Education 2017). Inwiefern Social Media bei der Personalisierung des Lernens als Vorbild angesehen wird und was es für die technische Umsetzung bedarf, wird in Kap. 3 dieses Beitrags näher erläutert.

\section{Potenziale technologischer Entwicklungen für das New Learning}

\subsection{Social-Media-based Learning}

Die Verwendung von technologischen Entwicklungen, die primär aus dem SocialMedia-Bereich bekannt sind, bieten für Lernangebote große Potenziale. Besonders die Nutzung einer an Social Media orientierten Plattformlösung soll im Folgenden näher betrachtet werden. Das interdisziplinäre Team aus Didaktikern und Wirtschaftsinformatikern leistet bei der Übertragung der vorhandenen Technologien auf den Bereich des Lernens einen entscheidenden Beitrag, um diese Potenziale auszuschöpfen. Die Didaktik beschäftigte sich hierbei primär mit dem methodisch sinnvollen Einsatz der Technologie (siehe Kap. 2), während die Wirtschaftsinformatik 
ihren Fokus auf die Optimierung der Mensch-Technik-Interaktion legt, die besonders im Bereich der Bildung eine zentrale Rolle einnimmt. Durch die Nutzung einer Plattformlösung wird die Verfügbarkeit des Lernangebots erhöht (siehe Kap. 2, Anforderungen an Ubiquitäres und situiertes Lernen). In traditionellen „,Vorort“-Kursen wird von den Lernenden verlangt, dass sie zu einer bestimmten Zeit an einem bestimmten Ort sind. Dieser Umstand schränkt Interessierte bereits vor Kursbeginn ein. Gegebenenfalls können sie nicht an den Veranstaltungen teilnehmen. Wenngleich die Möglichkeit besteht, Inhalte zu einem späteren Zeitpunkt nachzuholen, geht ihnen der potenzielle Austausch mit anderen Lernenden vor Ort verloren. Eine Plattform kann dieses Problem insoweit beheben, dass sowohl Zeit als auch Ort vom Lernenden frei gewählt werden können. Über dem Social-Media-Stil entsprechende Gruppen erhält der Lernende weiterhin die Möglichkeit, sich jederzeit mit anderen Kursteilnehmern und Lehrenden auszutauschen und somit seinen Lernerfolg zu erhöhen (siehe Kap. 2, Anforderungen an kollaboratives und gruppenzentriertes Lernen). Ein weiterer Vorteil ist die Vielfalt an Aufgaben, die auf einer digitalisierten Plattform bereitgestellt werden kann. Digitale Plattformen bieten dabei Ansatzpunkte für innovative Lernformate, wie beispielsweise interaktive Lehrvideos, progressive Lernpfade oder lernen in virtuellen Umgebungen mittels Virtual Reality-Brillen (siehe Kap. 2, Anforderungen an Kompetenz- und Handlungsorientiertes Lernen). Diese können die Zufriedenheit der Lernenden mit dem Angebot steigern und ihre Leistung verbessern (Thomas et al. 2018; Zhang et al. 2006). Weiterhin werden in traditionellen Kursen Aufgaben und Lernpfade meist für ganze Kursverbände erstellt. Somit muss jeder Lernende denselben Lernprozess durchlaufen und es wird keine Rücksicht auf persönliche Präferenzen und Stärken genommen. Auf einer digitalen Plattform besteht hingegen die Möglichkeit, verschiedene Varianten von Aufgaben zu erstellen, die speziell an Untergruppen von Lernenden angepasst wurden, um automatisiert individuelle Lernpfade für den Lernenden zu generieren (siehe Kap. 2, Personalisiertes und individualisiertes Lernen).

Die im Folgenden erläuterten Technologien sollen im Laufe der weiteren Entwicklung von School to go die Plattform weiter verbessern und können auch für andere Social-Media-basierte Lernplattformen große Potentiale bieten.

\subsection{Personalisierung als Schlüsselaspekt}

Die Personalisierung des Lernprozesses (siehe Kap. 2, Personalisiertes und individualisiertes Lernen) wird primär durch die Analyse der auf der Plattform erhobenen Daten ermöglicht. Durch die Vernetzung der Lernenden auf der Plattform entsteht ein an Social Media orientiertes Datennetzwerk. Lernende interagieren dabei mit Aufgaben, Foren, anderen Lernenden und mit Lehrenden. Während bei herkömmlichen Social-Media-Plattformen diese Daten ausgewertet und für die Personalisierung von Werbung verwendet werden, können im Rahmen einer Lernplattform die Daten zur Optimierung des Lernprozesses für den Lernenden sowie für andere Lernende genutzt werden. Von besonderer Bedeutung ist in diesem Hinblick der Begriff Learning Analytics (LA). Die Society for Learning Analytics Research definierte im Call for Papers der ersten Learning Analytics and Knowledge Conference im Jahr 2011 Learning Analytics als die Messung, Sammlung, Analyse und Berichterstattung von 
Daten über Lernende und ihre Kontexte zum Zwecke des Verständnisses und der Optimierung des Lernens und der Umgebungen, in denen es stattfindet (Society for Learning Analytics Research o. J.).

Die Verwendung von Predictive Learning Analytics (PLA) versucht im Gegensatz zur herkömmlichen LA aus den aktuellen und historischen Daten der Lernenden die Zukunft zu prognostizieren und somit den Lernprozess zu optimieren. Beispielhaft hierfür sind die Prognosen einer Abschlussnote oder die Bewertung einer Aufgabe durch den Lernenden anzuführen (ECAR-Analytics Working Group 2015; Shacklock 2016).

\subsection{Nutzung von Empfehlungssystemen}

Im Hinblick auf die Prognose der Bewertung einer Aufgabe durch den Lernenden gewinnen besonders Empfehlungssysteme als eine Ausprägung von PLA für die Optimierung des Lernprozesses an Relevanz. Empfehlungssysteme werden im Marketing schon lange als effektives Werkzeug eingesetzt. Eine Vielzahl von Kunden in Unternehmen wie Amazon oder Netflix werden dabei regelmäßig mit Empfehlungssystemen unterstützt. Bei Amazon ist es „Kunden, die diesen Artikel kaufen, kauften auch" während es bei Netflix weitere Filmempfehlungen sind (Lops et al. 2011; Schafer et al. 1999).

Mit Bezug auf die Auswertung der Daten der Lernplattform sollen drei Formen von Empfehlungssystemen näher betrachtet werden: kollaborative, inhaltsbasierte und hybride Empfehlungssysteme.

Systeme, die einen kollaborativen Ansatz verfolgen, vergleichen dabei die Bewertungen eines Lernenden bezüglich der Aufgaben mit denen ähnlicher anderer Lernender (Desrosiers und Karypis 2011; Schafer et al. 1999).

Besonders die Umsetzung einer Lernplattform im Social-Media-Stil unterstützt dieses Vorgehen, da durch die Netzwerkeffekte der Vergleich zwischen Lernenden vereinfacht wird und somit akkuratere Prognosen getroffen werden können. Ein inhaltsbasiertes Vorgehen nutzt einen anderen Ansatz. Es betrachtet lediglich die Attribute der vom spezifischen Lernenden bereits bearbeiteten Aufgaben und prognostiziert die Bewertung noch nicht bearbeiteter Aufgaben anhand ähnlicher Attribute (Desrosiers und Karypis 2011; Schafer et al. 1999).

Ein beispielhaftes Vorgehen hierfür wäre, die Nutzenden einzelne Aufgaben mit Schlagwörtern beschreiben zu lassen (tagging). Häufig von verschiedenen Lernenden genannte Tags werden die Aufgabe hierbei präziser beschreiben. Anschließend werden über einen Algorithmus noch nicht bearbeitete Aufgaben mit ähnlichen Tags gesucht, wie jene, die vom Nutzenden bereits gut bewertet wurden. Dieses Vorgehen nutzt im Gegensatz zum kollaborativen System die Netzwerkeffekte nicht aus, bietet allerdings den Vorteil, dass es auch bei geringer Nutzendenanzahl, z. B. in der Anfangsphase einer Plattform, präzise Ergebnisse liefern kann.

Das hybride Empfehlungssystem kombiniert die anderen beiden Vorgehensweisen. Dabei bestehen mehrere Möglichkeiten, die Empfehlungen der Systeme zu kombinieren, indem beispielsweise die unterschiedlichen Herangehensweisen jeweils gewichtet und zu einem Gesamtergebnis zusammengeführt werden. Eine weitere 
Möglichkeit ist nach einer festgelegten Bedingung, z.B. Anzahl der Bewertungen, das eine oder das andere System zu verwenden (Burke 2002).

Diese Systeme können nun genutzt werden, um den Lernpfad eines Lernenden zu optimieren (siehe Kap. 2, Personalisiertes und individualisiertes Lernen). Zum einen können dem Lernenden primär Aufgaben vorgeschlagen bzw. zugeordnet werden, die ihm vermutlich gefallen, um ihn im Kurs zu halten. Bei vorbestimmtem Curriculum kann eine Summe an Aufgaben zu bestimmten Themen mit demselben Inhalt bereitgestellt werden. Aus einer Gruppe von Aufgaben erhält der Lernende nun zu jedem Thema nur die Aufgabe, welche ihm voraussichtlich am besten gefallen wird.

Im Hinblick auf den Social-Media-Stil der Plattform ist auch ein weiteres Vorgehen mittels Empfehlungssystemen denkbar. Anstelle von Lerninhalten werden andere Lernende als potenzielle Lernpartner vorgeschlagen (siehe Kap. 2, Anforderungen an kollaboratives und gruppenzentriertes Lernen). Als Basis hierfür können zum einen die Merkmale der Lernenden verwendet werden, um z. B. Lernende mit komplementären Fähigkeiten oder Lernende mit ähnlichen Aufgabenpräferenzen zusammenzuführen. Zum anderen kann für die Bewertung herangezogen werden, wie gut ähnliche Lernende zusammengearbeitet haben. Die Bewertung dieser Zusammenarbeit kann dabei explizit, beispielsweise durch eine Punktvergabe durch die Lernenden, oder implizit, beispielsweise durch Überprüfung, ob die Lernenden in der Zukunft weiter zusammengearbeitet haben, umgesetzt werden.

\subsection{Bubble-Bildung im Social-Media-based Learning}

Ein Problem, welches in Social Media häufig auftritt und auch für eine Lernplattform nicht zu vernachlässigen ist, ist die sogenannte „Bubble-Bildung“. Gemeint ist hiermit, dass die Personen durch die Empfehlung von Inhalten, die sie voraussichtlich mögen, immer ähnliche Inhalte gezeigt bekommen und nicht mit neuen konfrontiert werden. Auf herkömmlichen Social-Media-Plattformen tritt dieses Problem häufig mit Bezug auf Nachrichten auf, sodass Meinungspluralismus durch den Algorithmus unterdrückt wird (Pariser 2011; Spohr 2017).

Ähnliches ist auf eine Lernplattform zu übertragen: Wenn Lernenden nur Aufgaben $\mathrm{zu}$ einem bestimmten Thema empfohlen werden, ist es ohne äußeren Einfluss unwahrscheinlich, dass er sich mit einem anderen Thema, welches ihm potenziell auch gefallen könnte, auseinandersetzt.

Eine Studie von Nguyen et al. hingegen zeigt, dass „Bubbles“ sowohl mit als auch ohne den Einsatz von Empfehlungssystemen entstehen. Die Empfehlungen, so das Ergebnis der Studie, fördern dabei die Bildung der „Bubble“ nicht, sondern verlangsamen sie. Da Empfehlungsalgorithmen personalisiert sind, wirken sich Benutzendenaktionen auf ihre Ausgabe aus. Items, die dem Nutzenden empfohlen und anschließend bewertet wurden, schienen die Vielfalt der zukünftig empfohlenen Items zu erweitern und nicht zu beschränken (Nguyen et al. 2014).

Das Phänomen der „Bubble-Bildung“ kann potenziell weiter unterdrückt werden, indem z.B. entgegen dem Empfehlungssystem immer wieder auch Aufgaben aus anderen Themenbereichen vereinzelt empfohlen werden, um ein Feedback des Nutzenden zu erhalten. 


\section{School to go}

\subsection{Hintergründe zur Initiative und Plattform}

Als Best-Practice Beispiel für eine Lernplattform im Social-Media-Stil soll im Folgenden die "School to go“ vorgestellt werden. Hierbei handelt es sich um eine gemeinsame Non-Profit-Initiative des Forschungsinstituts Bildung Digital (FoBiD) an der Universität des Saarlandes, des DFKI-Forschungsbereich Smart Enterprise Engineering und der Didactic Innovations GmbH. Mit der gemeinnützigen Initiative leisten die Institutionen einen Beitrag für eine nachhaltige digitale Bildung im Schulkontext. Die Zielgruppe der Angebote, die sich auf der gleichnamigen Plattform schooltogo.de befinden, sind gleichermaßen Schülerinnen und Schüler, Eltern, Lehrkräfte sowie die interessierte Öffentlichkeit. In Abb. 2 ist die Oberfläche der Plattform abgebildet, die durch den Aufbau und das Kachel-Design an gängige Social-Media-Kanäle erinnert und damit konkret auf die Bedürfnisse der Zielgruppe eingeht.

Ein interdisziplinäres Team aus Didaktikern und Wirtschaftsinformatikerin arbeitet seit den pandemiebedingten Schulschließungen im März 2020 kontinuierlich an dieser Plattform, um täglich neue motivierende und lehrplankonforme Inhalte für jedes Fach und jede Jahrgangsstufe bereitstellen zu können. Neben den eigens für "School to go" initiierten Neuproduktionen werden auch externe Lernformate im Angebotspool aufgenommen, die alle vor ihrer Veröffentlichung einer mehrstufigen Qualitätsprüfung unterzogen werden. Geprüft werden sie von einem aus Didaktikern und Technologen bestehenden Expertengremium anhand eines speziell für „School to go" entwickelten Kriterienkatalogs, der im Vorfeld gemeinsam von den beteiligten Institutionen vor dem Hintergrund der Qualitätssicherung entwickelt wurde.

Die Grundlage dieses Instruments bilden verschiedene Ausschluss- sowie Qualitätskriterien, die unterschiedliche Bereiche tangieren. Im Folgenden werden zehn dieser Kriterien (in Anlehnung an Goertz 2020) exemplarisch aufgeführt:

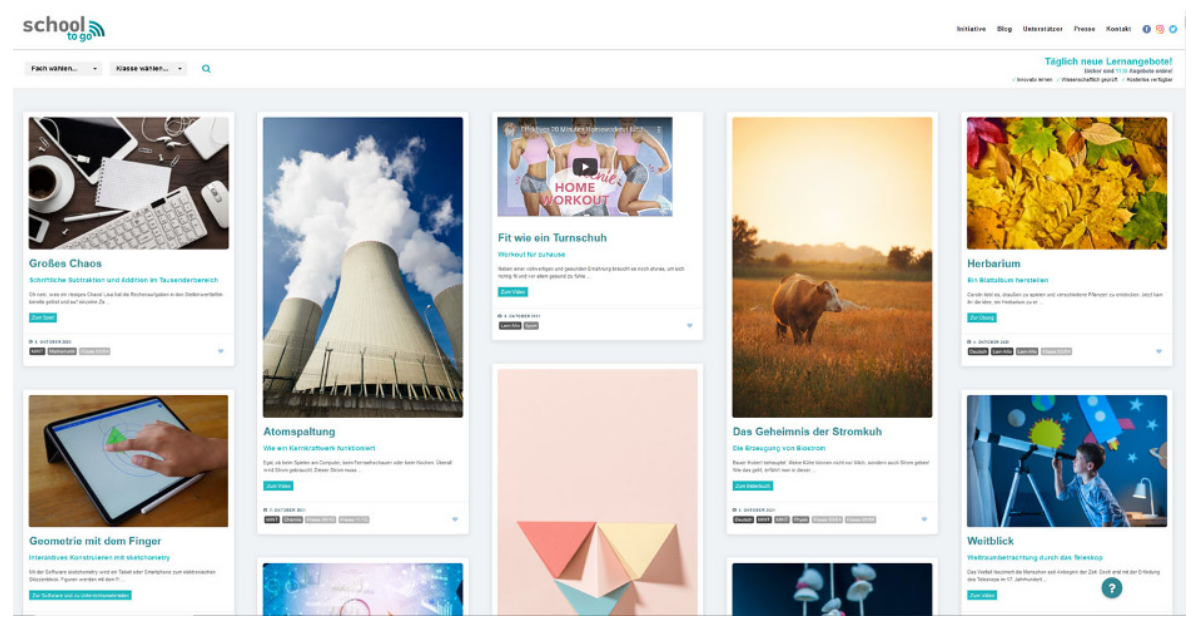

Abb. 2 Oberfläche der Plattform „School to go“ 
1. Inhaltliche Kriterien (u.a. fachliche Korrektheit und Aktualität des Lernangebots, Verständlichkeit aller relevanten Informationen)

2. Klarheit und Zielsetzung (u.a. Verständliche Darstellung der Zielsetzung des Lernangebots, ggf. unter Angabe von detaillierten Kompetenzniveaus)

3. Methodisch-didaktische Aufbereitung (u. a. didaktisch angemessene Darstellung der Informationen für die angegebene Zielgruppe)

4. Rolle und Aktivität der Lernenden (u. a. Einbezug der Lernenden durch Interaktivität, abwechslungsreiche Instruktionen sowie Sprachhandlungen)

5. Designumsetzung und Gestaltung (u. a. Attraktivität und visuelle Gestaltung des Lernangebots)

6. Technikeinsatz (u. a. technologische Korrektheit, Intuitivität der Techniknutzung)

7. Lernbegleitung (u.a. motivierende Lernbegleitung, konstruktives und positiv verstärkendes Feedback)

8. Selbstreguliertes Lernen und institutionelle Integrationsfähigkeit (u. a. Eignung zum selbstregulierten Lernen, flexible Integrierbarkeit der Lernangebote in den Schulalltag bzw. in einzelne Unterrichtsphasen)

9. Transparenz und Kosten-Nutzen-Verhältnis (u. a. transparente Darstellung der Finanzierung sowie aller anfallenden Kosten für die User)

10. Externe Evaluation (u. a. Bewertung des Angebots durch die Lernenden selbst, z. B. über Rezensionen und Kommentare)

Neben den Lernangeboten selbst finden sich unter der Rubrik „Blog“ in regelmäBigen Abständen neue Textbeiträge zu aktuellen Trendthemen der digitalen Bildung (z. B. „Bots für die Bildung“, „Mit Gamification die Lernmotivation steigern“ etc.). Als informelles Kommunikationsmedium beinhalten die gelisteten Blogbeiträge häufig auch Positionierungen und Aussagen von Bildungsexperten, die zu kontrovers diskutierten Themen im Kontext von Schule und Lernen Stellung beziehen. Bei der Ausgestaltung der Beiträge wird ein persönlicher und offener Sprachstil gewählt, der sich an einer breiten öffentlichen Zielgruppe orientiert. Über das integrierte Kontaktformular haben die User die Möglichkeit, Präferenzen für zukünftige Beiträge anzugeben und so das Angebot der Beiträge aktiv mitzugestalten.

\subsection{Exemplarisches Beispiel eines innovativen Lernformats}

Im Zeitalter der Digitalisierung verbreiten sich Meldungen mit rasanter Geschwindigkeit. In den Sozialen Medien werden sie von Usern häufig unreflektiert geteilt und so einer großen Community zugänglich gemacht. Eine Thematisierung des Phänomens der Falschmeldungen im schulischen Kontext ist erforderlich, um Kinder und Jugendliche schon frühzeitig für diese digitale Form der Meinungsmache zu sensibilisieren. Auf schooltogo.de findet sich daher unter dem Titel „Vorsicht: Fake! Falschnachrichten auf der Spur" ein interaktives Medienquiz (SWR Fakefinder Kids), bei dem die Lernenden in verschiedenen Levels entscheiden müssen, ob es sich in dem jeweiligen Beispiel um eine Form von Meinungsmache handelt. In Level 1 steht das Thema Werbung im Fokus, in dem Kindern und Jugendlichen Auszüge aus realen Social-Media-Stories von prominenten Persönlichkeiten präsentiert werden. Auf Basis des jeweiligen Beispiels stufen sie das Video in einen 
Beitrag mit oder ohne Werbeabsicht ein - eine Entscheidung, die je nach Fallbeispiel aufgrund von versteckter Produktplatzierung nicht immer einfach zu treffen ist. Belgleitet wird das Quiz von den beiden animierten Identifikationsfiguren Mona und Henri, die Feedback zu den jeweiligen Einordnungen geben und sich mit konkreten Erläuterungen und Hilfestellungen an die Lernenden richten. Level 2 nimmt sich dem Thema der Bildfälschungen an, denn nicht nur Bilder, sondern auch Videos lassen sich mithilfe digitaler Bearbeitungssoftware ohne große Aufwände und Vorkenntnisse manipulieren. Auch in diesem Level werden den Lernenden verschiedene Videoclips präsentiert, die sie auf Ihre Echtheit prüfen und in die entsprechenden Kategorien „Bildtrick“ oder „Kein Bildtrick“ einordnen müssen. Nach jedem abgeschlossenen Level besteht die Möglichkeit, entweder zum nächsten Level fortzuschreiten oder aber das aktuelle Level erneut zu durchlaufen. Während Level 3 das Thema Kettenbriefe anschaulich und interaktiv beleuchtet, finden sich in Level 4, dem sogenannten Superlevel, Aufgaben zu allen vorherigen Netzphänomenen (Werbung, Bildfälschungen, Kettenbriefe), mit dem sich die neu erworbenen Kenntnisse final überprüfen lassen (Poulet 2019).

Ein Infobogen für Lehrkräfte, der ebenfalls auf der Seite zum kostenlosen Download verlinkt ist, stellt neben der Arbeit im Selbststudium auch eine institutionelle Integrationsfähigkeit (siehe 4.1) des Medienquiz sicher. In Form einer Handreichung erhalten Lehrende konkrete Vorschläge für eine didaktisch motivierte Integration des Lernformats in den eigenen Unterrichtsverlauf. Begleitende Hinweise zu den einzelnen Netzphänomenen sowie Linklisten zu weiterführenden Informationen runden das Zusatzangebot ab.

\section{Zusammenfassung und Ausblick}

Ein Jahr nach der Veröffentlichung von „School to go“ befinden sich mehr als 1000 innovative Lernangebote auf der ersten Lernplattform im Social-Media-Stil. Mit dem steigenden Angebot wird sie in naher Zukunft eine stärkere Fächerdifferenzierung erhalten, sodass Lehrende und Lernende nicht nur nach Lernbereichen (MINT, Fremdsprachen etc.), sondern nach konkreten Fächern (z.B. Physik, Chemie etc.) filtern können. Über das Kontaktformular gehen zudem in regelmäßen Abständen eine Vielzahl von Anfragen zur Integration von User Generated Content ein. Diese von Usern selbst erstellten Inhalte reichen von Empfehlungslisten bis hin zu konkret ausgearbeiteten digitalen Lernmaterialien, die der interessierten Öffentlichkeit auch weiterhin kostenlos zur Verfügung stehen soll. Auch in Zukunft soll die Partizipation und Mitbestimmung der Nutzenden einen hohen Stellenwert bei ,School to go“ einnehmen, um so sicherzustellen, dass sich die Angebote an den tatsächlichen Interessen der Nutzenden orientieren und das Lernen auf der Plattform als sinnhaft erlebt wird.

Funding Open Access funding enabled and organized by Projekt DEAL.

Open Access Dieser Artikel wird unter der Creative Commons Namensnennung 4.0 International Lizenz veröffentlicht, welche die Nutzung, Vervielfältigung, Bearbeitung, Verbreitung und Wiedergabe in 
jeglichem Medium und Format erlaubt, sofern Sie den/die ursprünglichen Autor(en) und die Quelle ordnungsgemäß nennen, einen Link zur Creative Commons Lizenz beifügen und angeben, ob Änderungen vorgenommen wurden.

Die in diesem Artikel enthaltenen Bilder und sonstiges Drittmaterial unterliegen ebenfalls der genannten Creative Commons Lizenz, sofern sich aus der Abbildungslegende nichts anderes ergibt. Sofern das betreffende Material nicht unter der genannten Creative Commons Lizenz steht und die betreffende Handlung nicht nach gesetzlichen Vorschriften erlaubt ist, ist für die oben aufgeführten Weiterverwendungen des Materials die Einwilligung des jeweiligen Rechteinhabers einzuholen.

Weitere Details zur Lizenz entnehmen Sie bitte der Lizenzinformation auf http://creativecommons.org/ licenses/by/4.0/deed.de.

\section{Einhaltung ethischer Richtlinien}

Interessenkonflikt J. Eckle, A. Jungfleisch, D. Stattkus, N. Zarvić, J. Knopf und O. Thomas geben an, dass kein Interessenkonflikt besteht.

Ethische Standards Für diesen Beitrag wurden von den Autoren keine Studien an Menschen oder Tieren durchgeführt. Für die aufgeführten Studien gelten die jeweils dort angegebenen ethischen Richtlinien.

\section{Literatur}

ARD/ZDF-Forschungskommission (2020) ARD/ZDF-Onlinestudie. https://www.ard-zdf-onlinestudie.de/ files/2020/2020-10-12_Onlinestudie2020_Publikationscharts.pdf. Zugegriffen: 26. Mai 2021

Bendel O (2021) Soziale Medien. https://wirtschaftslexikon.gabler.de/definition/soziale-medien-52673. Zugegriffen: 4. Juni 2021

Burke R (2002) Hybrid recommender systems: survey and experiments. User Model User-Adapt Interact 12(4):331-370. https://doi.org/10.1023/A:1021240730564

Deges F (2018) Quick guide influencer marketing. Springer, Wiesbaden, S 33-41

Department of Education (2017) National education plan. https://tech.ed.gov/files/2017/01/NETP17.pdf. Zugegriffen: 30. Mai 2021

Desrosiers C, Karypis G (2011) A comprehensive survey of neighborhood-based recommendation methods. In: Ricci F, Lior R, Bracha S (Hrsg) Recommender systems handbook. Springer, Boston, S 107-144 https://doi.org/10.1007/978-0-387-85820-3_4

ECAR-Analytics Working Group (2015) The predictive learning analytics revolution: leveraging learning data for student success. ECAR working group paper

Eickelmann B, Drossel K (2020) Schule auf Distanz. Perspektiven und Empfehlungen für den neuen Schulalltag. Eine repräsentative Befragung von Lehrkräften in Deutschland. https://www.vodafonestiftung.de/umfrage-coronakrise-lehrer/. Zugegriffen: 30. Mai 2021 (Vodafone Stiftung Deutschland)

Goertz L (2020) Qualitätssicherung multimedialer Lernangebote. In: Niegemann H, Weinberger A (Hrsg) Handbuch Bildungstechnologie. Springer, Berlin, Heidelberg, S 481-491

Graf N, Schmitz AP (2020) Agiles Lernen, New Learning, Lernen 4.0. http://mentus.de/wp-content/ uploads/2020/01/Schmitz-Graf2020Agiles-Lernen-New-Learning-Lernen-4.0.pdf. Zugegriffen: 28. Mai 2021

Hubspot (2021) Social media trends. https://f.hubspotusercontent00.net/hubfs/53/Germany/offer_ files/Talkwalker\%20\%E2\%80\%93\%20Social\%20Media\%20Trends\%202021/Social_Trends_ Talkwalker-FINAL\%20-\%20DE.pdf?hubs_post-cta=author\&hubs_offer=offers.hubspot.de $\% 2$ Fsocial-media-trends-2021\&hubs_post=blog.hubspot.de\%2Fmarketing $\% 2$ Fsocial-mediain-deutschland\&submissionGuid=3d73cfb3-9758-4f5d-bf2a-c01ef13c92bb. Zugegriffen: 26. Mai 2021

Jahnke M (2021) Influencer Marketing - eine Bestandsaufnahem. In: Influencer Marketing. Springer, Wiesbaden, S 1-22

Kartes E et al (2020) Wie Lernen ein unsichtbarer Teil des Alltags wird. didacta 02:60-65

Kleinjohann M, Reinecke V (2020) Marketingkommunikation mit der Generation Z. Springer, Wiesbaden, S $1-6$ 
Lops P, De Gemmis M, Semeraro G (2011) Content-based recommender systems: state of the art and trends. In: Ricci F, Lior R, Bracha S (Hrsg) Recommender systems handbook. Springer, Boston, S 73-105 https://doi.org/10.1007/978-0-387-85820-3_3

Nguyen TT et al (2014) Exploring the filter bubble: the effect of using recommender systems on content diversity. Proceedings of the 23rd International Conference on World Wide Web, S 677-686 https:// doi.org/10.1145/2566486.2568012

Pariser E (2011) The filter bubble: how the new personalized web is changing what we read and how we think. Penguin Books, London

Poulet C (2019) Fakefinder Kinds. Infobogen für Lehrkräfte. https:/www.swr.de/unternehmen/ medienkompetenz/fakefinder-kids-infobogen-100.pdf. Zugegriffen: 26. Mai 2021

Schafer JB, Konstan J, Riedl J (1999) Recommender systems in e-commerce. Proceedings of the 1st ACM Conference on Electronic Commerce, S 158-166 https://doi.org/10.1145/336992.337035

Shacklock X (2016) From bricks to clicks. The potential of data and analytics in higher education. Higher Education Commission, London

Society for Learning Analytics Research (o. J.) What is learning analytics? https://www.solaresearch.org/ about/what-is-learning-analytics/. Zugegriffen: 19. Mai 2021

Spieler B (2006) Ansätze situierten Lernens in der (wirtschaftsberuflichen) Lehrerbildung. https://www. sowi-online.de/reader/lehrerausbildung_oekonomische_bildung/theorie_situierten_lernens.html. Zugegriffen: 28. Mai 2021

Spohr D (2017) Fake news and ideological polarization: filter bubbles and selective exposure on social media. Bus Inf Rev 34(3):150-160. https://doi.org/10.1177/0266382117722446

Statistisches Bundesamt (2020) Private Nutzung von Informations- und Kommunikationstechnologien. https://www.destatis.de/DE/Themen/Gesellschaft-Umwelt/Einkommen-KonsumLebensbedingungen/IT-Nutzung/Tabellen/internetaktivitaeten-personen-alter-ikt.html. Zugegriffen: 21. Mai 2021

Thomas O, Metzger D, Niegemann H, Welk M, Becker T (2018) GLASSROOM - Kompetenzaufbau und -entwicklung in virtuellen Lebenswelten. In: Thomas O, Metzger D, Niegemann H (Hrsg) Digitalisierung in der Aus- und Weiterbildung. Springer Gabler, Berlin, Heidelberg, S 2-19 https://doi.org/ 10.1007/978-3-662-56551-3_1

Zhang D, Zhou L, Briggs R, Nuanmaker J Jr (2006) Instructional video in e-learning: assessing the impact of interactive video on learning effectiveness. Inf Manag 43(1):15-27. https://doi.org/10.1016/j.im. 2005.01.004 